\title{
Circulating atrial natriuretic peptide genetic association study identifies a novel gene cluster associated with reduced NT-proANP, increased stroke and higher diastolic blood pressure
}

\author{
Naveen L Pereira, Nirubol Tosakulwong, Christopher G Scott, Gregory D Jenkins, Naresh Prodduturi, Yubo Chai, \\ Timothy M Olson, Richard J Rodeheffer, Margaret M Redfield MD, Richard M Weinshilboum, John C Burnett Jr.
}

From 7th International Conference on cGMP Generators, Effectors and Therapeutic Implications

Trier, Germany. 19-21 June 2015

\section{Introduction}

The goal of this study was to identify genetic determinants of plasma $\mathrm{N}$-terminal proatrial natriuretic peptide (NT-proANP) in the general community by performing a large-scale genetic association study and to assess its functional significance in in vitro cell studies and on disease susceptibility.

\section{Methods and results}

Genotyping was performed across 16,000 genes in 893 randomly selected individuals, with replication in 891 subjects from the community. Plasma NT-proANP1-98 concentrations were determined using a radioimmunoassay. Thirty-three genome-wide significant singlenucleotide polymorphisms were identified in the MTHFR-CLCN6-NPPANPPB locus and were all replicated. To assess the significance, in vitro functional genomic studies and clinical outcomes for carriers of a single-nucleotide polymorphism rs5063 (V32M) located in NPPA that represented the most significant variation in this genetic locus were assessed. The rs5063 variant allozyme in transfected HEK293 cells was decreased to $55 \pm 8 \%$ of wild-type protein $(\mathrm{p}=0.01)$ as assessed by quantitative western blots. Carriers of rs5063 had lower NT-proANP levels (1427 versus $2291 \mathrm{pmol} / \mathrm{L} ; \mathrm{P}<0.001)$ and higher diastolic blood pressures $(75$ versus $73 \mathrm{~mm}$ $\mathrm{Hg} ; \mathrm{p}=0.009$ ) and were at an increased risk of stroke when compared with wild-type subjects independent of age, sex, diabetes mellitus, hypertension, atrial fibrillation, and cholesterol levels (hazard ratio, 1.6; $\mathrm{p}=0.004$ ).

\section{Conclusion}

This is the first large-scale genetic association study of circulating NT-proANP levels performed with replication and functional assessment that identified genetic variants in the MTHFR-CLCN6-NPPA-NPPB cluster to be significantly associated with NT-proANP levels. The clinical significance of this variation is related to lower NT-proANP levels, higher blood pressures, and an increased risk of stroke in the general community.

Published: 2 September 2015

doi:10.1186/2050-6511-16-S1-A37

Cite this article as: Pereira et al:: Circulating atrial natriuretic peptide genetic association study identifies a novel gene cluster associated with reduced NT-proANP, increased stroke and higher diastolic blood pressure. BMC Pharmacology and Toxicology 2015 16(Suppl 1):A37.

\footnotetext{
* Correspondence: burnett.john@mayo.edu

Cardiorenal Research Laboratory, Division of Cardiovascular Diseases, and Department of Health Sciences Research, Mayo Clinic, Rochester, MN 55905, USA
} 